\title{
La décentralisation : un enjeu stratégique pour le Québec
}

\author{
Guy Massicotte \\ Université du Québec
}

\section{Introduction}

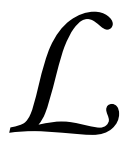
e but de cet article est de démontrer que la décentralisation des pouvoirs publics est un enjeu stratégique pour le Québec et de montrer que, puisqu'il en est un, il devrait se traduire en stratégies à mettre en œuvre en fonction de critères de succès.

\section{Pourquoi décentraliser?}

Pourquoi décentraliser? Cette question est souvent posée. Elle est parfois formulée d'une façon naïve, comme au premier degré : est-ce nécessaire, est-ce bien utile ? Parfois aussi, elle est posée d'une façon plus problématique, elle vise alors les raisons qui militent en faveur d'une plus grande décentralisation.

La principale équivoque met en cause la motivation même des protagonistes car, sous les apparences de la recherche d'une plus grande performance ou d'une plus grande équité, la volonté de décentralisation peut renvoyer aussi au désengagement de l'État, à

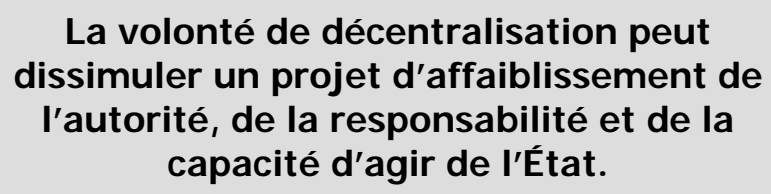

La volonté de décentralisation peut dissimuler un projet d'affaiblissement de l'autorité, de la responsabilité et de la capacité d'agir de l'État.

Laissées à elles-mêmes, les forces économiques créent du développement qui n'est pas toujours dans les formes souhaitées, d'où la nécessité des politiques publiques et des interventions gouvernementales. Il y a là une polarisation entre forces économiques et action publique. Les pouvoirs publics interviennent, par exemple, pour défendre les droits des consommateurs, protéger les travailleurs ou l'environnement, éviter la concentration excessive des entreprises dans un secteur d'activité, etc. La décentralisation peut être un moyen de contrecarrer la métropolisation perçue comme un effet pervers du marché. Mais elle peut être, aussi, une façon d'affaiblir l'État et donc de l'affaiblissement des structures politiques et à une place plus importante laissée aux forces du marché.

\section{La place du politique}

La question qui se pose au point de départ est celle de la place même du politique. Faut-il laisser aux phénomènes économiques et sociaux le soin de fixer le destin des sociétés, ou y a-t-il place pour une action politique, pour une volonté délibérée d'une autorité légitime visant à influer sur le cours des choses et y parvenant à l'occasion?

Cette question est importante, car la volonté de décentralisation peut dissimuler un projet d'affaiblisse- renforcer les forces du marché et la concentration des ressources.

\section{Forces du marché et territoire}

Par rapport au territoire, les forces du marché vont dans le sens de la métropolisation, mais, pour ce qui est du rôle des politiques publiques, les choses sont plus complexes. Il n'y a pas de consensus clair sur les bienfaits ou les méfaits de la métropolisation. Tous sont d'accord pour protéger les consommateurs, les travailleurs, l'environnement. Les seules restrictions viennent de la conviction qu'une trop grande protection pourrait nuire à l'économie, en rendant les entreprises moins concurrentielles. Mais par rapport 
au territoire, il s'en trouve pour vanter les mérites de la métropolisation du point de vue économique, forçant les opposants à se placer sur un autre plan, social, politique ou éthique. Même au nom du développement économique, personne n'approuve l'exploitation des travailleurs ou celle des consommateurs ou la dégradation de l'environnement, mais il s'en trouve pour approuver la métropolisation.

Plus encore, chacun sait que les forces de marché sont souvent soutenues par les politiques publiques, comme c'est le cas par exemple avec la métropolisation, dans le contexte de la concurrence internationale à laquelle sont confrontés les grands pôles urbains. Dès lors, il existe une ligne de fracture entre les politiques publiques qui secondent les forces du marché et celles qui cherchent à contrecarrer des tendances jugées néfastes ou qui proposent d'autres voies de développement.

\section{Le projet de décentralisation ne vise pas à affaiblir l'État, son but est d'élargir la base économique du Québec.}

\section{La décentralisation, facteur de développement économique}

En faisant l'hypothèse, toutefois, que la décentralisation puisse favoriser l'économie de marché, à l'instar de certaines mesures sociales qui paraissent restrictives à première vue et qui finissent par être bénéfiques à l'économie de marché, comme les mesures de protection des travailleurs qui favorisent la productivité ou les mesures de protection de l'environnement qui engendrent de nouvelles opportunités économiques, on obtient un autre cas de figure. La décentralisation, en renforçant les capacités d'action sur un plus vaste territoire, ne peut-elle pas contribuer à élargir les bases mêmes de l'économie de marché, contribuer à diminuer les charges des zones plus développées et permettre d'ajouter à la richesse globale ?

\section{La décentralisation, facteur d'efficience des politiques publiques}

L'administration des politiques publiques, avec l'ajustement constant qu'elles impliquent à l'échelle des territoires, constitue une charge pour l'économie et la société. Le degré de centralisation et de décentralisa- tion constitue une variable significative, la décentralisation pouvant être considérée comme une source de charges supplémentaires ou, au contraire, comme une source d'efficacité et d'efficience.

\section{En somme...}

Les motivations et les objectifs d'un projet de décentralisation ne sont pas aussi univoques qu'ils puissent paraître de prime abord.

- La décentralisation correspond-elle à l'affaiblissement ou au renforcement du rôle de l'État et de l'influence des citoyens sur l'économie et la société ?

- La décentralisation vise-t-elle à mieux soutenir les forces du marché, en affaiblissant l'État, ou à les harnacher, en contrecarrant la métropolisation considérée comme un effet pervers de l'évolution économique laissée à elle-même ?

- La décentralisation nuit-elle à l'économie en contrecarrant la métropolisation considérée alors comme un facteur de succès du développement économique?

- La décentralisation favorise-t-elle l'élargissement de la base économique considérée comme un facteur de succès du développement économique?

- La décentralisation contribue-t-elle à l'accroissement des charges publiques ou, au contraire, estelle une source d'efficience et d'efficacité des politiques publiques?

Nous faisons l'hypothèse que le projet de décentralisation ne vise pas à affaiblir l'État, qu'il constitue au contraire une manière de lui permettre de mieux jouer son rôle de développer le Québec, selon la volonté démocratiquement exprimée des citoyens. Nous faisons l'hypothèse qu'il vise à contrecarrer les effets pervers du marché que constitue la métropolisation et que, ce faisant, il n'est pas nuisible à l'économie puisque son but est d'élargir la base économique du Québec en favorisant une répartition plus équilibrée des personnes, des emplois, des infrastructures, de la richesse et des possibilités de développement sur l'ensemble du territoire. Nous faisons l'hypothèse, enfin, que le projet de décentralisation ne constitue pas une charge additionnelle, mais qu'il permet, au contraire, d'atteindre une plus grande efficience et une plus grande efficacité des politiques publiques. 


\section{Quoi décentraliser?}

De prime abord, il paraît pertinent d'élaborer un cadre de réflexion pour guider le choix des pouvoirs et des responsabilités qui auraient avantage à être décentralisés.

\section{Soutenir l'innovation}

Une idée directrice qui devrait inspirer le projet de décentralisation devrait être l'innovation, le soutien de la capacité d'innovation des acteurs dans chaque territoire du Québec. Il faut, à cet égard, dépasser le soutien à l'innovation sectorielle et soutenir l'innovation territoriale, ce qui exige l'élaboration et la mise en œuvre d'une véritable stratégie territoriale d'innovation.

La maîtrise d'œuvre d'une telle stratégie requiert des ressources importantes en termes de légitimité et d'autorité politiques, en termes de ressources financières et en termes d'expertise, de capacités d'action humaines et professionnelles. Il faut renforcer les instances locales pour créer les conditions nécessaires à l'exercice d'un pouvoir véritable sur l'économie et la société.

L'évolution récente des politiques publiques convie à cette approche. Le gouvernement du Québec s’appuie de plus en plus sur les acteurs locaux et régionaux pour l'élaboration et la mise en œuvre des politiques de développement économique. Il s’agit d'une évolution positive. Le développement passe par l'engagement de chaque milieu, des entrepreneurs et des acteurs publics dans toutes les sphères d'activités : infrastructures, ressources naturelles, éducation, santé, science et technologie, loisir, culture, environnement, etc.

L'enjeu, dès lors, devient de renforcer les milieux pour leur permettre de jouer ce rôle, et de le faire de manière à ce qu'ils s'engagent dans une stratégie délibérée de soutien à l'innovation.

Cela passe par le renforcement de l'autorité publique à l'échelle locale et par la dévolution de responsabilités et de ressources. Concrètement, les leviers plus directement au cœur de l'innovation devraient être décentralisés : outils de transfert et d'innovation en lien avec les universités et les collèges, ressources de formation professionnelle et de développement de la main-d'œuvre, aide aux entreprises, moyens reliés à l'amélioration du cadre de vie, développement durable, environnement, santé, culture, loisir, etc.

\section{Renforcer l'efficience et l'efficacité des politiques publiques}

Une autre idée directrice concerne la nécessité d'accroître l'efficience et l'efficacité des politiques publiques. Le Québec est parvenu à un point de saturation fiscale, alors que les besoins continuent toujours de croître. Le transfert démographique vers une population plus âgée ne fera qu'aggraver ce problème. Seule une plus grande productivité des organisations, notamment dans le service public, pourra permettre de maintenir les politiques en les finançant de façon adéquate.

\section{Une idée directrice qui devrait inspirer le projet de décentralisation devrait être l'innovation, le soutien de la capacité d'innovation des acteurs dans chaque territoire du Québec.}

Il faut faire ici le pari que le rapprochement des besoins et des moyens constitue une source d'efficience et d'efficacité. Que ce soit dans des domaines comme l'organisation de l'enseignement primaire, les soins de santé de première ligne, les services à la petite enfance et aux aînés, le développement de la maind'œuvre, le soutien à l'innovation et au transfert technologique, l'aide aux entreprises, le développement durable, la protection de l'environnement, les loisirs, la culture, la sécurité des personnes, etc., les interventions publiques ont tout avantage à être prises en charge, conçues, développées et mises en œuvre par des pouvoirs locaux bien au fait des besoins et des préférences des citoyens. Le moins paradoxal, à cet égard, n'est pas de constater que ce fait est déjà largement reconnu puisque l'État a souvent créé des structures locales et régionales pour administrer des politiques sectorielles en éducation, en santé ou dans le développement économique.

Un premier pas dans la voie de la décentralisation serait de regrouper autour d'une instance politique unifiée dans l'axe municipalités-MRC les pouvoirs et responsabilités déjà institués sur le territoire. Un 
second pas serait d'accroître le panier de responsabilités et de ressources existant à l'échelle locale, notamment les champs qui touchent plus directement les citoyens et qui ont un impact sur la capacité d'action et d'innovation d'un milieu. Un troisième pas serait d'orienter leur développement en fonction de la prise en charge et du soutien à l'innovation.

\section{Une autre idée directrice concerne la nécessité d'accroître l'efficience et l'efficacité des politiques publiques.}

\section{Le cercle vertueux - pour une vision intégrée du développement local}

Plus de ressources humaines et financières, plus de compétences disponibles, plus de pouvoirs et de responsabilités, des politiques publiques mieux adaptées, plus efficientes et plus efficaces, une bonne gouvernance du système territorial d'innovation, plus de développement économique, autant de moyens se soutenant mutuellement. Pour instituer ce cercle vertueux, au moins quatre conditions sont nécessaires :

- Implanter à l'échelle locale le maximum de compétences et de ressources financières : transférer des postes et des enveloppes financières, autant que possible en réaménageant la fiscalité et en mettant en place des programmes de péréquation pour les milieux plus démunis. Prévoir aussi des ressources d'accompagnement pour les milieux moins bien pourvus en compétences professionnelles et techniques.

- Confier à une autorité politique unique, imputable démocratiquement, la responsabilité de l'ensemble des politiques et des moyens décentralisés à l'échelle locale. C'est là, tout simplement, un enjeu de renforcement du pouvoir et de la capacité d'intervention de l'autorité publique qui est en cause, une autorité qui devrait nécessairement être située dans l'axe municipalités-MRC, et dont la forme précise serait à établir selon les conditions locales, principalement géographiques et démographiques.

- Créer un encadrement d'appui à l'échelle nationale, promouvoir et soutenir, dans chaque milieu, une stratégie de développement axée sur la gouver- nance de l'innovation par la mobilisation de toutes les ressources du milieu.

Une telle stratégie n'affaiblit pas l’État. Elle lui permet de mieux jouer son rôle. Elle ne requiert pas non plus de ressources additionnelles, sinon celles qu'un milieu voudra se donner dans le cadre du processus démocratique. Une telle stratégie ne divise pas le Québec. Elle conduit à l'élargissement de sa base économique. Sa mise en œuvre, toutefois, ne va pas de soi et ses architectes devraient s'inspirer de l'analyse stratégique et tenir compte de facteurs de succès.

\section{Comment décentraliser ? Analyse stratégique et facteurs critiques de succès}

Pour réussir la décentralisation, il y aurait lieu de s’inspirer de l'approche du changement stratégique et structurel. Elle concerne des changements fondamentaux considérés comme importants, voire décisifs.

La mise en œuvre du changement stratégique suppose qu'on analyse certains paramètres qui servent d'emprise à des facteurs critiques de succès : la clarification des enjeux et la vision de changement; la prise en compte du système d'acteurs et du contexte d'action, l'arrimage de la vision, du système d'acteurs, du contexte, des objectifs et des moyens, l'importance du suivi et de l'évaluation.

Un premier facteur de succès est de mieux préciser les motifs qui inspirent le projet de décentralisation et les objectifs qui le sous-tendent. Il suppose que les acteurs adhèrent à une vision, comme celle énoncée précédemment, débarrassée d'équivoques et d'ambiguïtés.

Deux facteurs de succès concernent les ressources du système d'action : le système d'acteurs et le contexte d'action, en l'occurrence le contexte de la décentralisation.

\section{Les ressources du système d'action : le système d'acteurs}

Le projet de décentralisation s’inscrit dans un système d'action qui en délimite les possibilités.

L'analyse stratégique devrait permettre de clarifier les motivations de grands groupes d'acteurs, de préciser 
les positions de chacun, de révéler les changements à apporter pour neutraliser les oppositions et de déceler les potentiels d'alliance et de compromis. Bien connaître les ressources du système d'acteurs et les exploiter de manière à renforcer les appuis et en réduire les oppositions constitue un deuxième facteur de succès.

L'analyse révèle la faiblesse de la base du pouvoir politique en faveur de la décentralisation.

- Les tenants des forces du marché devraient être pour la décentralisation dans la mesure où ils l'assimilent à un affaiblissement de l'État, mais deux raisons les en empêchent :

- la plupart estiment que l’État doit soutenir les forces gagnantes et n’ont pas vraiment intérêt à affaiblir l’État;

- comme la décentralisation pourrait servir à équilibrer les tendances à la métropolisation, ils n’ont aucun intérêt à la soutenir.

- Les tenants du leadership et de l'autorité du politique sont gênés de soutenir la décentralisation, dans la mesure où elle est perçue comme un affaiblissement de l’État.

Cette structure est à l'œuvre dans l'ensemble du territoire, même en région, où beaucoup d'acteurs craignent que la décentralisation amène l'affaiblissement de l'État et implique son désengagement vis-à-vis des régions plutôt que le renforcement du pouvoir régional et de ses capacités d'action. Ils considèrent souvent que l'État est le seul frein à la métropolisation et que son affaiblissement conduira à encore plus de métropolisation.

Cette analyse commande des approches politiques convergentes avec la vision des enjeux :

- la décentralisation n’est pas contraire aux forces du marché, elle vise à élargir la base économique du Québec et, à terme, elle profitera aussi bien aux métropolitains qu'aux régionaux;

- la décentralisation ne vise pas ni ne conduit à l'affaiblissement de l'État ou à son renforcement, mais elle permet, au contraire, d'optimiser l'action de l'État, de rendre les politiques publiques plus efficaces et plus efficientes.

Il importe pour les protagonistes de la décentralisation de tenir compte du système d'action et de s'y inscrire de manière à mobiliser les forces du changement en sa faveur et de neutraliser ainsi les forces d'opposition.

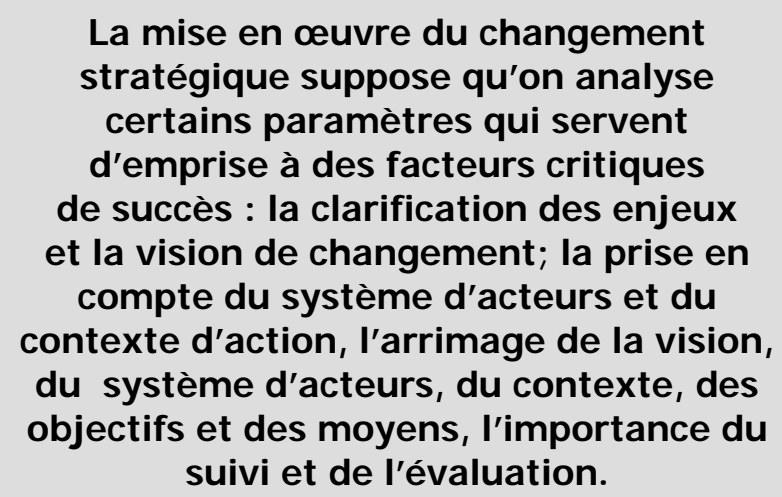

D’une part, il faudrait que le projet de décentralisation s'appuie sur les forces économiques implantées à l'extérieur de la métropole. Certes, les forces du marché conduisent à la métropolisation, perçue comme un phénomène incontournable qu'il faut soutenir dans une perspective de concurrence internationale. Mais il s'agit là, en bonne partie, d'une vue de l'esprit. Il existe dans tous les territoires des acteurs économiques qui agissent dans le marché et qui auraient tout à gagner à des politiques de soutien mieux adaptées à leur situation. Il suffit de visiter le Québec pour découvrir la richesse du tissu économique qui ne demande qu'à être mieux soutenu non pas contre la métropole, mais pour lui-même.

D’autre part, il faudrait que le projet de décentralisation devienne un projet d'amélioration de l'État, non pas plus d'État mais « mieux d'État », c'est-à-dire une manière d'organiser l'État pour le rendre plus efficient et plus efficace. La décentralisation doit devenir un projet d'État et non un projet contre l'État. Il est clair qu'un groupe particulier d'acteurs, soit les administrateurs publics, doit être rallié à cette vision.

Plus fondamentalement, la stratégie doit tenir compte de l'environnement, c'est-à-dire de la situation économique, sociale, culturelle dans laquelle elle s'inscrit. 


\section{Les ressources du système d'action : le contexte de la décentralisation}

Il importe de bien connaître le contexte d'action pour élaborer et mettre en œuvre une stratégie gagnante. La prise en compte du contexte constitue un troisième facteur de succès.

Il existe plusieurs éléments de contexte qui sont favorables à la décentralisation. La décentralisation est un concept qui affecte les politiques publiques un peu partout dans le monde. Ce mouvement correspond à la double volonté d'optimiser les politiques et les ressources publiques et d'instrumenter les collectivités locales (empowerment). Le consensus est assez large qui constate que le développement économique s'élabore dans des milieux innovants, sectoriels parfois, mais souvent territoriaux, à l'échelle d'une région, voire d'une microrégion.

Un autre élément de contexte favorable à la décentralisation est la complexité même de la gestion des politiques publiques dans la société actuelle et la difficulté concomitante de mettre sur pied des politiques adaptées à la diversité des situations particulières. L'optimisation des politiques publiques est un enjeu lié à cette complexité. Elle exige la décentralisation à l'échelle locale des politiques et des programmes pour qu'ils soient adaptés aux situations particulières par les acteurs qui ont une connaissance directe et pointue de ces situations.

Mais à côté de ces facteurs positifs, il en existe de moins favorables qui exigent d'être pris en compte. Le traumatisme du délestage en est un. Il y a eu plusieurs expériences dans le passé où la décentralisation s'est traduite par un délestage de responsabilités au palier local, sans ressources supplémentaires adéquates. Cette expérience a créé un traumatisme qui sert de repoussoir, ou tout au moins de prétexte aux opposants. Tout projet de décentralisation devra tenir compte de ces convictions et se réaliser dans un contexte de transparence, avec des conditions susceptibles de restaurer la confiance des acteurs locaux.

Mais il existe d'autres contraintes plus fondamentales. Il faut tout d'abord noter l'immensité du territoire québécois et la faible densité de la population à l'extérieur de la grande région montréalaise. L'étendue du territoire a provoqué la création de deux struc- tures d'organisation, la région et la MRC ou ce qui en tient lieu. Dans certains cas, la région est trop vaste, ce qui a amené, au cours des années, à créer de nouvelles régions. Dans d'autres cas, la MRC est ellemême trop fractionnée entre plusieurs municipalités de taille, de fonctions et d'intérêts variés. Les réformes récentes des structures municipales ont permis de progresser en éliminant bon nombre d'irritants et en favorisant l'adaptation des structures aux réalités socio-économiques et sociopolitiques de chaque milieu.

Il reste que la coexistence d'une double structure constitue une contrainte majeure à la décentralisation qui, d'une certaine façon, a été renforcée par la création des CRÉ (Conférences régionales des élus). On comprend bien que, dans la mesure où la région devient un palier politique, il est difficile qu'elle échappe à l'autorité politique des élus. Mais fallait-il en faire un palier politique ? Si l'on veut un projet viable de décentralisation, il faudra trancher dans le vif à cet égard. La région, comme région administrative, peut être un lieu de déconcentration des pouvoirs publics, mais non pas un lieu de décentralisation, à moins d'en faire un gouvernement régional, ce qui n’apparaît pas une voie réaliste. Politiquement, le palier visé par la décentralisation doit être le milieu local et régional, dans l'axe municipalités-MRC. Ce qui n'empêche pas de réaliser des mises en commun, des regroupements de services et de fonctions à l'échelle régionale, mais la responsabilité politique ne peut pas être située au palier régional, au sens de la région administrative ou économique.

À cet égard, la réforme des structures gouvernementales pourrait favoriser la clarification du contexte si elle aboutissait à distinguer clairement la stratégie économique de l'État et la décentralisation des pouvoirs publics à l'échelle locale. Les régions, tout comme le palier local, deviennent des cadres d'action du gouvernement, qui s'appuie sur les CRÉ, sur les structures municipales, sur les CLD (Centres locaux de développement) pour l'élaboration de plans de développement économique, de politiques, de programmes de soutien. Dans une logique d'action gouvernementale, rien n'empêche, bien au contraire, que cette action soit menée en concertation, voire en collaboration, avec les milieux concernés, en leur laissant le maximum d'autonomie. Mais il ne faudrait pas parler, à cet égard, de décentralisation. La décentralisation devrait être conçue comme une politique gouverne- 
mentale visant à renforcer les pouvoirs des gouvernements locaux.

Il faudrait maintenant définir des indicateurs qui permettent d'apprécier, à long terme, le développement des capacités d'innovation et de l'innovation elle-même à l'échelle des territoires. II faudrait, aussi, mesurer l'efficience et l'efficacité des politiques publiques aussi bien par rapport à la qualité de vie des citoyens, et à leur volonté démocratiquement exprimée, que par rapport à leur impact sur le développement.

Une autre dimension du contexte qui interfère fortement avec le projet de la décentralisation est l'inégalité des ressources fiscales et des capacités d'action des citoyens. Une véritable décentralisation doit nécessairement s'accompagner de la responsabilité fiscale, soit de la maîtrise d'une assiette fiscale propre et pleinement maîtrisée par l'autorité politique qui exerce les responsabilités. Or, comment décentraliser, en assurant une certaine forme d'égalité dans l'accès aux services publics de tous les citoyens, si les moyens sont à ce point disparates d'un endroit à l'autre du territoire ? S'il n'y avait que la dimension fiscale, le problème pourrait assez facilement se résoudre par des mécanismes adéquats de péréquation, mais il y a aussi la capacité, la volonté, dans certains cas, d'assumer de nouveaux pouvoirs et de nouvelles responsabilités. Pour tenir compte de ces éléments de contexte, tout projet de décentralisation devrait comporter, en même temps que le transfert de ressources fiscales, un programme de péréquation pour assurer l'égalité dans la disponibilité des ressources. Il devrait aussi comporter des mécanismes d'accompagnement, voire d'option, une sorte de décentralisation à la carte en somme, tout au moins au cours d'une phase de transition.

\section{Vers un projet de décentralisation bien maîtrisé}

Un quatrième facteur de succès est l'arrimage étroit qui doit exister entre les objectifs et les modalités de mise en œuvre du projet, d'une part, et les enjeux, le système d'action et le contexte, de l'autre. Le projet de décentralisation esquissé précédemment correspond à cette exigence. D'autant plus que bien intégré, il a le potentiel d'instituer un cercle vertueux de développement. On peut alors parler d'un cinquième facteur de succès qui consiste à agencer les approches de façon à générer un cercle vertueux d'actions de développement qui se soutiennent elles-mêmes.

\section{Conclusion : pilotage et évaluation}

La mise en œuvre d'un projet de décentralisation appelle l'instauration de mécanismes de suivi et d'évaluation qui permettent la rétroaction et l'ajustement des objectifs et des moyens aux enjeux, au système d'action et au contexte, en temps réel et de façon continue. Si le suivi de la mise en œuvre des moyens implique plus simplement la vérification sur le terrain de la réalisation effective des mesures prévues, l'évaluation de l'atteinte des objectifs suppose l'élaboration d'indicateurs de réussite.

Dans ce texte, nous avons défini les objectifs à la lumière d'une vision clarifiée des enjeux, des ressources et des contraintes du système d'action et des éléments de contexte, autour de deux grandes pistes : l'innovation et la performance des politiques publiques.

En toute logique, il faudrait maintenant définir des indicateurs qui permettent d'apprécier, à long terme, le développement des capacités d'innovation et de l'innovation elle-même à l'échelle des territoires. Il faudrait, aussi, mesurer l'efficience et l'efficacité des politiques publiques aussi bien par rapport à la qualité de vie des citoyens, et à leur volonté démocratiquement exprimée, que par rapport à leur impact sur le développement, soit, en fin de compte, sur l'innovation elle-même, dans l'esprit même du cercle vertueux qui devrait demeurer la finalité ultime de l'ensemble de cette démarche.

L’instauration de mécanismes de suivi et d'évaluation qui assurent la réussite à long terme du processus de changement constitue à cet égard un sixième facteur de succès. 


\section{Publicité}

«Quelle est votre opinion » 